Heightened disgust propensity and contamination fear in OCD - 1

\title{
Is Heightened Disgust Propensity Truly a Risk Factor for
}

Contamination-Related Obsessive-Compulsive Disorder?

\author{
Gabriele Melli
}

Institute for Behavioral and Cognitive Psychology and Psychotherapy of Florence (IPSICO)

University of Pisa

Andrea Poli

Institute for Behavioral and Cognitive Psychology and Psychotherapy of Florence (IPSICO)

Carlo Chiorri

University of Genova

Bunmi O. Olatunji

Vanderbilt University 
Heightened disgust propensity and contamination fear in OCD - 2

\begin{abstract}
Disgust propensity (DP) has been conceptualized as a stable personality trait that confers risk for contamination-related OCD (C-OCD). However, the extent to which DP leads to the subsequent development of C-OCD is unclear. In fact, the presence of C-OCD might lead to an increase in DP rather than the inverse. The present study was aimed to test this hypothesis in a large clinical sample of OCD patients ( $\geq 21$ years of age) with (C-OCD; $n=56)$ and without (NC-OCD; $n=103$ ) contamination-related symptoms that completed measures of OCD symptoms, depression, anxiety, and DP. DP was assessed twice, in reference to the present situation (T1) and to when the participant was 18 years old (T0). The two groups did not significantly differ in DP at T0. However, C-OCD participants reported higher DP scores than NC-OCD at T1. Furthermore, the T1 vs T0 difference in DP was significant only in the C-OCD group. Subsequent analyses also showed that T1 DP levels, but not T0 levels, significantly predicted contamination-related symptoms and that contamination-related symptoms significantly predicted T1 DP levels when reversing the variables. Despite study limitations, these findings question the role of DP as a risk factor for C-OCD.
\end{abstract}

Keywords: obsessive-compulsive disorder, OCD, disgust propensity, disgust, contamination fear 
Is heightened disgust propensity truly a risk factor for contamination-related obsessive-compulsive disorder?

\section{Introduction}

Obsessive compulsive disorder (OCD) is characterized by the occurrence of persistent thoughts, urges, or images that are experienced as intrusive and unwanted (i.e., obsessions), and compulsive actions that the individual feels driven to perform in response to an obsession or according to rules that must be applied rigidly aimed at preventing or reducing anxiety or distress, or preventing some dreaded event or situation (DSM-5; American Psychiatric Association, 2013). A common obsession in OCD relates to fear of contamination (Summerfeldt, Antony, Downie, Richter, \& Swinson, 1997). According to Rachman's cognitive model, obsessive thoughts about contamination elicit irrational fears that induce compulsive behaviours aimed at neutralizing them, and avoidance of the supposed contaminated substances (Rachman, 2004). However, developments in theory (Woody \& Teachman, 2000) and research (Woody \& Tolin, 2002) suggest that, in addition to irrational fear, the emotion of disgust likely plays a central role in obsessive thoughts regarding uncleanliness, contamination, and dirt that is commonly observed in contamination-based OCD (C-OCD; Brady, Adams, \& Lohr, 2010; Power \& Dalgleish, 1997).

Disgust propensity (DP) - individual differences in the perceived frequency/intensity of experiencing disgust - is a personality trait that has been implicated in the development and maintenance of OCD symptoms, in particular when they are related to contamination fear (David et al., 2009; Olatunji, Sawchuk, Lohr, \& de Jong, 2004; Olatunji, Williams, Lohr, \& Sawchuk, 2005; Schienle, Stark, Walter, \& Vaitl, 2003). Indeed, several studies have supported the link between DP and OCD. For example, cross-sectional research has shown that self-report measures of DP positively correlate with self-report measures of contamination fear (Mancini, Gragnani, \& D’Olimpio, 2001; Moretz \& McKay, 2008; Olatunji et al., 2004, 2007; Thorpe, Patel, \& Simonds, 2003). Moreover, other studies have found that the relationship between DP and OCD symptoms remains when controlling for negative affect (Cisler, Olatunji, \& Lohr, 2009; Melli, Bulli, Carraresi, 
\& Stopani, 2014; Melli, Chiorri, Carraresi, Stopani, \& Bulli, 2015a; Melli et al., 2015c; Olatunji et al., 2007). In fact, neurobiological studies have showed that brain regions involved in disgust processing (i.e., insula, ventrolateral prefrontal cortex) appear to play a role in C-OCD (Husted, Shapira, \& Goodman, 2006; Lawrence et al., 2007). There is clearly a converging literature implicating DP in C-OCD and this literature suggests that avoidance of disgust is one of the main motivational dimensions of contamination fear that is distinct from harm avoidance (Melli et al., 2017b; Melli, Chiorri, Carraresi, Stopani, \& Bulli, 2015b; Rouel, Stevenson, \& Smith, 2018).

Despite the demonstrated link between DP and contamination/washing symptoms in OCD, it remains unclear if heightened DP causes the development of C-OCD. Longitudinal research in nonclinical samples has attempted to address this question. For example, David and colleagues (2009) found that individual differences in disgust did not significantly predict residual change in total symptoms of OCD over a 12-week period when controlling for risk factors for anxiety disorder symptoms in general (e.g., negative affect, anxiety sensitivity) and OCD specifically (e.g., obsessive beliefs). However, exploratory analyses did suggest that individual differences in disgust may be predictive of residual change in some OCD symptom (i.e., hoarding) subtypes but not others (i.e., washing). In contrast, Olatunji (2010) found that change in disgust levels over a 12-week period predicted change in symptoms of contamination-based OCD, even when controlling for age, gender, and change in negative affect. However, this association was driven by change in the perceived negative impact of experiencing disgust rather than change in the intensity that disgust is generally experienced. A subsequent longitudinal study found that changes in DP between baseline and a six-month follow-up assessment were associated with changes in overall self-reported OCD symptoms but not with changes in contamination-based OCD symptoms (Berle et al., 2012).

Treatment outcome research has shown that change in DP is significantly associated with improvement in contamination/washing symptoms in patients with OCD (Athey et al., 2015; Knowles, Viar-Paxton, Riemann, Jacobi, \& Olatunji, 2016). In fact, Olatunji, Tart, Ciesielski, McGrath and Smits (2011b) found that decreases in DP over time during exposure-based treatment 
mediated improvement in OCD symptoms, even after controlling for improvements in negative affect. Although treatment outcome research suggests that targeting DP during treatment may result in improvements in OCD symptoms, longitudinal research has provided mixed evidence on the extent to which DP causes the development of OCD. Although DP has traditionally been conceptualized as a trait-like personality characteristic, Olatunji (2015) found that selective engagement in health-related behaviors significantly increased DP. This finding suggests that DP is indeed malleable and an alternative hypothesis may be that the presence of OCD symptoms leads to an increase in DP, or at least that DP and OCD symptoms influence each other in a reciprocal fashion similar to the effect observed with other trait-like variables (e.g. anxiety sensitivity) and disorder symptoms (e.g., PTSD; Marshall, Miles, \& Stewart, 2010).

The present study was hence aimed to build on the existing literature by further clarifying the temporal relationship between DP and OCD symptoms in a large clinical sample. More specifically, a retrospective longitudinal design was employed to examine the relationships between current contamination-related symptoms and both current DP levels (in reference to the next few days, T1) and retrospectively assessed DP levels (in reference to when the participant was 18 years old, T0), controlling for anxiety and depression, in a sample of OCD patients with (C-OCD) and without (NC-OCD) contamination concerns. Specifically, we aimed to investigate whether (a) COCD patients showed higher DP levels than NC-OCD at T1 but not at T0; (b) C-OCD patients only showed higher DP levels at T1 than at T0; (c) DP at T1, but not at T0, predicted contamination fear symptoms in C-OCD patients, after controlling for anxiety and depression; and (d) contamination fear symptoms predicted DP at T1 in C-OCD patients, after controlling for anxiety and depression.

\section{Method}

\section{Participants}

A total of 172 OCD patients was referred to an Italian private adult psychotherapy center for evaluation and treatment. During the routine assessment phase, patients were interviewed by one of the members of the research team (all doctoral psychologists experienced in diagnosing psychiatric 
disorders) using the Italian versions of the Anxiety Disorder Interview Schedule IV (ADIS-IV; Brown, Di Nardo, \& Barlow, 1994) and the Yale-Brown Obsessive-Compulsive Scale-Second Edition (Y-BOCS-II; Storch et al., 2010; Italian version in Melli et al., 2015d) to establish diagnoses. Each case was audio-recorded, carefully reviewed during supervisory meetings and all diagnoses were reached by raters' consensus (inter-rater reliabilities were excellent: ADIS-IV: $k=$ .93 ; Y-BOCS-II: ICC $=.95$ ). Some participants had one or more secondary diagnoses, including anxiety disorders (social phobia $[n=8]$, panic disorder $[n=11]$ and generalized anxiety disorder $[n$ $=26]$ ) and mood disorders (major depressive disorder $[n=46]$ ). Potential participants with a secondary or tertiary diagnosis of OCD were excluded. Participants under 21 years of age and participants who reported an onset of the disorder before 20 years of age were also excluded as were those with psychosis, current mania, and/or substance dependence.

The final sample included 159 OCD patients. Participants who reported contamination-related symptoms or concerns as a primary complaint, as determined by the Y-BOCS-II $(n=56)$, were assigned to the OCD Contamination (C-OCD) sub-group; participants who met the diagnostic criteria for primary OCD, but who did not report contamination-related symptoms or concerns as a primary complaint ( $n=103$ ) were assigned to the Non-Contamination OCD (NC-OCD) sub-group. Demographic information about the samples is reported in Table 1.

[Table 1]

\section{Measures}

Disgust Propensity Questionnaire (DPQ; Melli, Chiorri, Stopani, Bulli \& Carraresi, 2017a). The DPQ is a 9-item measure to assess DP in Italian samples, as the Italian version (Melli, Chiorri, \& Smurra, 2013) of the Disgust Scale-Revised (DS-R; Olatunji et al., 2007) - the best-known scale for the assessment of DP - had shown satisfactory, but not excellent psychometric properties, and some of the items of this scale are not appropriate to the Italian cultural context. Participants are asked to rate each item on a five-point Likert scale from 0 ('not at all') to 4 ('very much'). This questionnaire was found to have a one-factor structure, very good internal consistency ( $\alpha$ in the 
range $.85-.91)$, adequate test-retest reliability $(\mathrm{ICC}=.85)$, and construct validity. In the present study, Cronbach's $\alpha$ was .89.

Dimensional Obsessive-Compulsive Scale (DOCS; Abramowitz et al., 2010). The DOCS is a 20-item scale that assesses the main OCD symptom dimensions: contamination obsessions as well as washing and cleaning compulsions; obsessions about responsibility for harm and checking compulsions; repugnant obsessive thoughts and mental compulsive rituals or other covert neutralizing strategies; obsessions about order and symmetry and ordering or arranging compulsions. Within each symptom dimension, items assess 5 severity parameters in relation to the past month and are rated on a scale ranging from 0 ('no symptoms') to 4 ('extreme symptoms'). The subscales were found to be highly valid and reliable (Abramowitz et al., 2010). The Italian version of the DOCS (Melli et al., 2015e) replicated the four-factor structure of the original version and showed good internal consistency ( $\alpha>.80$ for all subscales), adequate temporal stability (ICC > .75 for all scales), and good construct validity. In the present study, Cronbach's $\alpha$ was in the range .94-.97.

Beck Anxiety Inventory (BAI; Beck \& Steer, 1990). This is a 21-item self-report inventory that assesses the severity of state anxiety. Participants are asked to rate the severity of the symptoms described by the items a scale ranging from 0 ('not at all') to 3 ('severely'). The original version has shown good psychometric properties (Beck et al., 1988), and the Italian version has shown a onefactor structure, good internal consistency $(\alpha>.80)$, adequate test-retest reliability $(r>.62)$, and good construct validity (Sica \& Ghisi, 2007). In the present study, Cronbach’s $\alpha$ was .90 .

Beck Depression Inventory-II (BDI-II; Beck, Steer, \& Brown, 1996). This 21-item self-report inventory is used to assess depressive symptoms over the preceding two weeks. Items concern different depressive symptom domains, with four possible answers describing symptoms of increasing severity associated with a score 0 ('absent') to 3 ('severe'). The BDI-II has shown good psychometric properties (Beck et al., 1996) and the Italian version of the BDI-II (Sica \& Ghisi, 
Heightened disgust propensity and contamination fear in OCD - 8

2007) has shown a one-factor structure, adequate internal consistency ( $\alpha$ s in the range $.80-.87$ ), testretest reliability $(r=.76)$, and construct validity. In the present study, Cronbach's $\alpha$ was .92 .

\section{Procedure}

All participants volunteered to take part in the study after being introduced to a detailed description of the procedure. They signed a written informed consent form and were treated in accordance with the Declaration of Helsinki and the Ethical Principles of Psychologists and Code of Conduct (American Psychological Association, 2010). Participants completed the OCD, depression, and anxiety measures once, and the DPQ twice; one time was in reference to T1 (current DP) and another time was in reference to T0 (DP at age 18), in order to retrospectively assess DP before the disorder onset. All the measures were administered in a counterbalanced fashion to control for order and sequence effects, and batteries took between 10 and 20 minutes to be completed. DPQ instructions in reference to T1 were: "Below you will find some common hypothetical situations. Please rate how much disgust you would feel if the following situations happened to you in the next few days"; while DPQ instructions in reference to T0 were: "Please answer the same questions rating how much disgust you would have felt if these situations had happened to you when you were 18 years old. Clearly, your recollection may not be accurate, but please try to picture yourself at that time anyway and imagine being faced with such a situation. Don't be concerned with your previous answers, but focus on how your life was when you were about 18 years old". No external incentives were offered to participate in this study.

\section{Statistical analyses}

A mixed factorial ANOVA was performed on DPQ scores, with Time as the within-subjects factor and Group as the between-subjects factor. Post-hoc tests on the interaction effect allowed to test the hypotheses of significantly higher DPQ scores at T1, but not at T0, in the C-OCD sample with respect to the NC-OCD sample, and of significantly higher DPQ scores at T1 for the C-OCD sample, but not for the NC-OCD sample, with respect to T0. 
Zero order correlations between all of the measures were computed for the whole sample. A series of hierarchical multiple regression analyses was then performed in the C-OCD subsample to determine whether DPQ scores at $\mathrm{T} 1$, but not at $\mathrm{T} 0$, contributed to the prediction of $\mathrm{C}-\mathrm{OCD}$ symptoms above and beyond depression and anxiety, and conversely whether C-OCD symptoms at T1 were able to predict DPQ scores at T1, over and beyond anxiety and depression. The Variance Inflation Factor (VIF) was computed for each predictor and it always fell within the range (1.031.93) which is considered as evidence of a lack of substantial multicollinearity (Menard, 1995). Further examination of the data also indicated that the assumptions of linearity and homoscedasticity were met. Separate regressions were conducted using the DOCS-Contamination subscale or DPQ scores as dependent variables. In the first step (Model 1) of each regression model, BDI-II and BAI scores were entered as control variables. In the second step (Model 2) DPQ scores at T1, DPQ scores at T0, or DOCS-Contamination subscale scores were then entered to examine whether current and past DP could account for a further proportion of variance of C-OCD symptoms, when DOCS-Contamination subscale was the dependent variable, or if C-OCD symptoms could account for a further proportion of variance of DP, when DPQ scores at T1 was entered as the dependent variable.

\section{Results}

\section{Mean comparisons for $D P Q$ scores}

Table 2 shows mean comparisons of DPQ scores at T1 and at T0 between and within C-OCD and NC-OCD samples. All the effects of the mixed factorial ANOVA were statistically significant (Time: $F(1,157)=53.64, p<.001, \eta^{2}=.06$; Group: $F(1,157)=12.55, p=.001, \eta^{2}=.06$; Interaction: $\left.F(1,157)=55.72, p<.001, \eta^{2}=.06\right)$. Consistent with hypotheses, post-hoc tests showed that DPQ scores at T1 were significantly higher $(t(157)=9.19 ; p<.001, d=1.58)$ than those at T0 for the C-OCD sample, while DPQ scores at T1 were not significantly higher $(t(157)=$ $0.12 ; p=.906, d=0.02$ ) than those at T0 for the NC-OCD sample. Furthermore, DPQ scores at T1 
for the C-OCD sample were significantly higher $(t(157)=6.52 ; p<.001, d=1.12)$ than those for the NC-OCD sample, while DPQ scores at T0 for the C-OCD sample were not significantly higher $(t(157)=0.07 ; p=.941, d=0.01)$ than those for the NC-OCD sample.

An important consideration is that there was considerable variability in the amount of time that passed since participants were 18 years old. $(M=16.42, D S=9.42$, range 3-46). Accordingly, the comparison of DPQ scores at T1 and at T0 between and within C-OCD and NC-OCD samples was repeated with time passed since participants were 18 years also as a covariate. The main effect of time and of the covariate were not significant, while the other effects remained substantially unchanged. More importantly, the pattern of results of the post-hoc comparisons was the same.

[Table 2]

\section{Zero order correlations}

DPQ at T1 scores were strongly correlated with DOCS-Contamination scores at T1, while they showed a weak or non-significant correlations with all of the other measure scores at T1 (noncontamination OCD symptom severity, depression, and anxiety). DPQ at T0 scores were not significantly correlated with DOCS-Contamination scores at T1 and weakly or not significantly correlated with all of the other measure scores at $\mathrm{T} 1$.

\section{[Table 3]}

\section{Current contamination-related OCD symptoms predicted by current (T1) DP}

In the first step of the hierarchical multiple regression analysis predicting the DOCS-

Contamination subscale score, the BDI-II and BAI scores did not explain a significant proportion of variance $\left(R^{2}=.01 ; p>.05\right)$. In the second step, entering the DPQ scores at T1 significantly increased the variance explained $\left(R^{2}\right.$ change $\left.=.27 ; p<.001\right)$. In this model current DP emerged as a strong significant predictor $(\beta=.53 ; p<.001)$ of contamination-related symptoms when controlling for depression and anxiety.

Current contamination-related OCD symptoms predicted by DP at 18 years of age (TO) 
In the first step of the regression analysis predicting the DOCS-Contamination subscale score, the BDI-II and BAI scores did not explain a significant proportion of variance $\left(R^{2}=.01 ; p>\right.$ .05). In the second step, entering the DPQ scores at T0 did not significantly increase the variance explained $\left(R^{2}\right.$ change $\left.=.01 ; p>.05\right)$. DP when individuals were 18 years old did not emerge as a significant predictor of current contamination-related symptoms when controlling for depression and anxiety.

\section{[Table 4]}

\section{Discussion}

The present study examined the differential association between retrospectively recalled DP (T0) before the onset of OCD, and current (T1) DP and symptoms of C-OCD. Zero order correlations on the whole sample showed that DP levels at T1 were strongly correlated with contamination-related symptoms, while they were weakly or not significantly correlated with all of the other OCD symptom dimensions. For those participants with C-OCD, DP was observed to be significantly higher at T1 than at T0. However, for those with NC-OCD, DP at T0 did not significantly differ from DP at T1. Furthermore, DP scores at T1 for the C-OCD group were significantly higher than those for the NC-OCD sample, while DP scores at T0 for the C-OCD sample were not significantly higher than those for the NC-OCD sample.

The findings of the present study are largely consistent with previous research showing that when considering several OCD symptom groups (checking, ordering, and washing), the clearest relationship is between washing symptoms of OCD and DP (Tolin, Woods, \& Abramowitz, 2006). Compared to other variants of OCD, DP may be most robustly characteristic of C-OCD. Although previous research has shown that DP is elevated in a heterogeneous OCD sample when compared with patients with generalized anxiety disorder and with a non-clinical sample (Olatunji, Ebesutani, David, Fan, \& McGrath, 2011a), more recent research has also shown that DP is especially increased in C-OCD patients, but not in patients with checking-related OCD symptoms or in nonclinical participants (Garcìa-Soriano et al., 2016). However, it is important to note that the stronger 
link between DP and C-OCD compared to NC-OCD has not be consistently observed in the literature (David et al., 2009; Berle et al., 2012). Although the results of the present study contribute to the evidence for a relatively stronger relationship in the case of $\mathrm{C}-\mathrm{OCD}$, more methodologically rigorous research is needed to better characterized the link between DP and the heterogeneous symptoms of OCD.

The present findings also highlight important patterns in the time course of DP in C-OCD. Most notably is the finding that recalled DP levels (before OCD onset) appears to be similar for those with C-OCD and those with NC-OCD. This finding appears to be inconsistent with the view that heightened DP may confer risk for C-OCD (Olatunji et al., 2011a, 2011b). The finding that heightened DP was observed among those with C-OCD, relative to those with NC-OCD at T1 (after OCD onset), suggests that DP levels increases concurrently with the disorder. This finding is in contrast with the traditional view of DP as a stable personality trait and suggest that the construct have both time-varying and time-invariant components. This view is consistent with previous research showing that excessive engagement in health-related safety behaviors (i.e., hand washing) does significantly increase DP (Olatunji, 2015). These findings also raise the possibility that elevated DP in C-OCD may be a consequence rather than a cause of the disorder.

Regression analyses also showed that current DP, but not retrospectively assessed DP, predicted current C-OCD symptoms when controlling for anxiety and depression. It is important to note that in the first step of the hierarchical multiple regression predicting the DOCS-Contamination subscale score, the BDI-II and BAI scores did not explain a significant proportion of variance. This finding highlights that individual differences in anxiety and depression do not appear to be especially useful when explaining variance in symptoms of C-OCD. Rather, DP at T1 was observed to be a significant predictor of contamination-related symptoms when controlling for depression and anxiety. This finding is fully consistent with previous research showing that DP predicts symptoms of C-OCD (Mancini et al., 2001; Olatunji et al., 2004, 2007) even when controlling for various indicators of negative affect (Melli et al., 2015a; Olatunji et al., 2007). In contrast, retrospectively 
recalled DP (T0) did not emerge as a significant predictor of current contamination-related symptoms when controlling for depression and anxiety. This finding also calls into question the extent to which DP may be conceptualized as a risk factor for C-OCD and also suggests that retrospectively assessed DP levels per se are not sufficient to predict the emergence of clinically relevant C-OCD symptoms. Considering that exposure-based treatment significantly decreases both OCD symptoms and DP in adolescent (Knowles et al., 2016) and adult (Athey et al., 2015) samples, a more parsimonious account is that the presence of OCD and associated safety behaviors may be a learning context for an increase in DP.

The present study also found that concurrent DP was a significant predictor of DOCSContamination when controlling for depression and anxiety. Given the cross-sectional nature of this study, no conclusive causal inferences can be drawn from this finding. In fact, it is important to note that this finding does not necessarily imply that DP is a cause of contamination-related concerns, rather it suggests that DP and contamination concerns in OCD have a robust relationship that cannot be explained by associations with depression and anxiety. This finding is further evidence that concurrent levels of DP, but not general anxiety or depression, may serve as a unique indicator of C-OCD symptom severity. An alternative interpretation of these findings is that DP and C-OCD have a synergistic and reciprocal relationship where the presence of one (C-OCD) leads to an increase in the other (DP). This may be why it is commonly observed that as OCD improves during treatment so does DP (Athey et al., 2015; Knowles et al., 2016). This is consistent with the hypothesis that the presence of C-OCD may be a learning context where experiencing symptoms essentially hijacks other psychological processes, like DP, that may function to facilitate contaminants avoidance. This interpretation suggests that DP may be better conceptualized as a maintenance factor for C-OCD rather than a risk factor. Whereas a risk factor is a variable that has been shown to prospectively predict some subsequent pathological outcome, a maintenance factor predicts symptom persistence over time (Stice, 2002). Indeed, DP has been shown to predict 
concurrent contamination fear better than other psychological processes, like obsessive beliefs (Melli, Moulding, \& Poli, 2016).

Our findings must be considered in the context of some limitations. First, our study is retrospective and the reliance on retrospective self-reports may potentially introduce recall bias (cf. Field, Argyris, \& Knowles, 2001; King, Eleonora, \& Ollendik, 1998). The use of retrospective reports inevitably introduces a few sources of bias that can threaten the validity of the data (e.g., Sudman, Bradburn, \& Schawrz, 1996), especially when the intensity of a symptom has to be reported (as it is the case of the DPQ; Schwarz, 2007). Another important issue with this approach is that asking patients with contamination OCD to complete a measure of DP for now and for when they were 18 may set up the measures as a direct contrast with each other. In fact, even though all of the administered symptom measures were counterbalanced to reduce the risk of order effects, a second related limitation of the present study is that time 0 DPQ and the time 1 DPQ were administered on the same page and the latter always followed the former. This approach raises the possibility that participants' ratings of their time 0 items were influenced by recent ratings of time 1 items. Furthermore, current contamination concerns might make disgust more salient now compared to when they were 18 years old. Future research that employs longitudinal designs that prevents participants from conflating their OCD symptoms with DP will be needed to more directly examine the extent to which DP is a cause or consequence of OCD.

A third limitation is that participants were self-selected and this might limit the generalizability of our conclusions to the population of OCD patients. Fourth, DP is only one of the many variables that may contribute to C-OCD symptoms. Future research would benefit from the inclusion of measures of additional potential vulnerability factors for contamination symptoms, particularly mental contamination (e.g., the Vancouver Obsessional Compulsive Inventory - Mental Contamination Scale; Melli, Carraresi, Stopani, Radomsky, \& Bulli, 2015f; Radomsky, Rachman, Shafran, Coughtrey, \& Barber, 2014), as recent research provide support for its role in triggering contamination fear based upon disgust avoidance (Carraresi, Bulli, Melli, \& Stopani, 2013; Melli et 
al., 2014; Melli et al., 2017b). Fifth, a single measure of C-OCD was used in the present study. Future research will benefit from use of multiple C-OCD symptom measures from varying levels of analysis. Indeed, a sixth limitation of the present study is that all of the data was derived from selfreport measures which tends to inflate associations among variables. Future research addressing these limitations may further clarify the role of DP in OCD. Finally, participants were classified according to whether contamination concerns were their "primary complaint" on the Y-BOCS II. However, some of the patients $(n=14)$ in the NC-OCD group had contamination concerns as a secondary complaint. A more conservative analysis, excluding these patients from the NC-OCD group did not change the findings.

Despite these limitations, the present study highlights the important of future research designed to determine the extent to which DP may be conceptualized as a risk or maintenance factor for C-OCD as this may have important implications for treatment or prevention efforts. 


\section{References}

Abramowitz, J. S., Deacon, B. J., Olatunji, B. O., Wheaton, M. G., Berman, N. C., \& Losardo, D. (2010). Assessment of obsessive-compulsive symptom dimensions: development and evaluation of the Dimensional Obsessive-Compulsive Scale. Psychological Assessment, 22, 180-198. http://dx.doi.org/10.1037/a0018260

American Psychiatric Association (2013). Diagnostic and statistical manual of mental disorders (5th ed.). Washington, DC: Author.

American Psychological Association (2010). Ethical principles of psychologists and code of conduct. http://www.apa.org/ethics/code/principles.pdf.

Athey, A. J., Elias, J. A., Crosby, J. M., Jenike, M. A., Pope Jr., H. G., Hudson, J. I., \& Brennan, B. P. (2015). Reduced disgust propensity is associated with improvement in contamination/washing symptoms in obsessive-compulsive disorder. Journal of Obsessive-Compulsive and Related Disorders, 4, 20-24. http://dx.doi.org/10.1016/j.jocrd.2014.11.001

Beck, J. S., \& Steer, R. A. (1990). Beck Anxiety Inventory manual. San Antonio, TX: Psychological Corporation.

Beck, J. S., Steer, R. A., \& Brown, G. K. (1996). Manual for the Beck Depression Inventory-II. San Antonio,TX: Psychological Corporation. 
Berle, D., Starcevic, V., Brakoulias, V., Sammut, P., Milicevic, D., Hannan, A., \& Moses, K. (2012). Disgust propensity in obsessive-compulsive disorder: Cross-sectional and prospective relationships. Journal of Behavior Therapy and Experimental Psychiatry, 43, 656-663. http://dx.doi.org/ 10.1016/j.jbtep.2011.09.002

Brady, R. E., Adams, T. G., \& Lohr, J. M. (2010). Disgust in contamination-based obsessivecompulsive disorder: A review and model. Expert Review of Neurotherapeutics, 10, 1295-1305. http://dx.doi.org/10.1586/ern.10.46

Brown, T. A., Di Nardo, P., \& Barlow, D. H. (1994). Anxiety disorders interview schedule adult version (ADIS-IV):client interview schedule. Oxford: Oxford University Press.

Carraresi, C., Bulli, F., Melli, G., \& Stopani, E. (2013). Mental contamination in OCD: its role in the relationship between disgust propensity and fear of contamination. Clinical Neuropsychiatry, 10 (suppl. 1), 13-19. http://dx.doi.org/10.1521/ijct_2015_8_01

Cisler, J. M., Olatunji, B. O., \& Lohr, J. M. (2009). Disgust, fear, and the anxiety disorders: A critical review. Clinical Psychology Review, 29, 34-46. http://dx.doi.org/10.1016/j.cpr.2008.09.007

David, B., Olatunji, B. O., Armstrong, T., Ciesielski, B. G., Bondy, C. L., \& Broman-Fulks, J. (2009). Incremental specificity of disgust sensitivity in the prediction of obsessive-compulsive disorder symptoms: Cross-sectional and prospective approaches. Journal of Behavior Therapy and Experimental Psychiatry, 40, 533-543. http://dx.doi.org/10.1016/j.jbtep.2009.07.004

Field, A. P., Argyris, N. G., \& Knowles, K. A. (2001). Who's afraid of the big bad wolf: A prospective paradigm to test Rachman's indirect pathways in children. Behavior Research and Therapy, 39, 1259-1276. http://dx.doi.org/10.1016/S0005-7967(00)00080-2

García-Soriano, G., Rosell-Clari, V., Serrano, M. A. (2016). Emotional and Cognitive Variables Associated with Contamination-Related Obsessive-Compulsive Symptoms. Spanish Journal of Psychology, 19, E25. http://dx.doi.org/10.1017/sjp.2016.27. 
Husted, D. S., Shapira, N. A., \& Goodman, W. K. (2006). The neurocircuitry of obsessivecompulsive disorder and disgust. Progress in Neuro Psychopharmacology and Biological Psychiatry, 30, 389-399. http://dx.doi.org/10.1016/j.pnpbp.2005.11.024

Kahneman, D., Fredrickson, B. L., Schreiber, C. A., \& Redelmeier, D. A. (1993). When more pain is preferred to less: adding a better end. Psychological Science, 4(6), 401-405. http://dx.doi.org/10.1111/j.1467-9280.1993.tb00589.x

King, N. J., Eleonora, G., \& Ollendick, T. H. (1998). Etiology of childhood phobias: Current status of Rachman's three pathways theory. Behavior Research and Therapy, 36, 297-309. http://dx.doi.org/10.1016/S0005-7967(98)00015-1

Knowles, K. A., Viar-Paxton, M., Riemann, B. C., Jacobi, D. M., \& Olatunji, B. O. (2016). Is disgust proneness sensitive to treatment for OCD among youth? Examination of diagnostic specificity and symptom correlates. Journal of Anxiety Disorders, 44, 47-54. http://dx.doi.org/10.1016/j.janxdis.2016.09.011

Lawrence, N. S., An, S. K., Mataix-Cols, D., Ruths, F., Speckens, A., \& Phillips, M. L. (2007). Neural responses to facial expressions of disgust but not fear are modulated by washing symptoms in OCD. Biological Psychiatry, 61, 1072-1080. http://dx.doi.org/10.1016/j.biopsych.2006.06.033

Mancini, F., Gragnani, A., \& D’Olimpio, F. (2001). The connection between disgust and obsessions and compulsions in a non-clinical sample. Personality and individual Differences, 31, 11731180. http://dx.doi.org/10.1016/S0191-8869(00)00215-4

Marshall, G. N., Miles, J. N. V., \& Stewart, S. H. (2010). Anxiety sensitivity and PTSD symptom severity are reciprocally related: Evidence from a longitudinal study of physical trauma survivors. Journal of Abnormal Psychology, 119, 143-150. http://dx.doi.org/10.1037/a0018009

Melli, G., Avallone, E., Moulding, R., Pinto, A., Micheli, E., \& Carraresi, C. (2015d). Validation of the Italian version of the Yale-Brown Obsessive Compulsive Scale-Second Edition (Y-BOCS- 
Heightened disgust propensity and contamination fear in OCD - 19

II) in a clinical sample. Comprehensive Psychiatry, 60, 86-92. http://dx.doi.org/10.1016/j.comppsych.2015.03.005

Melli, G., Bulli, F., Carraresi, C., \& Stopani, E. (2014). Disgust propensity and contaminationrelated OCD symptoms: The mediating role of mental contamination. Journal of ObsessiveCompulsive and Related Disorders, 3, 77-82. http://dx.doi.org/10.1016/j.jocrd.2014.01.002

Melli, G., Bulli, F., Carraresi, C., Tarantino, F., Gelli, S., \& Poli, A. (2017b). The differential relationship between mental contamination and the core dimensions of contact contamination fear. Journal of Anxiety Disorders, 45, 9-16. http://dx.doi.org/10.1016/j.janxdis.2016.11.005

Melli, G., Carraresi, C., Stopani, E., Radomsky, A., \& Bulli, F. (2015f). Factor structure and temporal stability of the Vancouver Obsessional Compulsive Inventory - Mental Contamination Scale (VOCI-MC) and psychometric properties of its Italian version. Comprehensive Psychiatry, 58, 198-204. http://dx.doi.org/10.1016/j.comppsych.2014.12.017

Melli, G., Chiorri, C., Bulli, F., Carraresi, C., Stopani, E., \& Abramowitz, J. S. (2015e). Factor Congruence and Psychometric Properties of the Italian Version of the Dimensional ObsessiveCompulsive Scale (DOCS) Across Non-Clinical and Clinical Samples. Journal of Psychopathology and Behavioral Assessment, 37, 329-339. http://dx.doi.org/10.1007/s10862014-9450-1

Melli, G., Chiorri, C., Carraresi, C., Stopani, E., \& Bulli, F. (2015a). The role of disgust propensity and trait guilt in OCD symptoms: A multiple regression model in a clinical sample. Journal of Obsessive-Compulsive and Related Disorders, 5, 43-48. http://dx.doi.org/ 10.1016/j.jocrd.2015.01.007

Melli, G., Chiorri, C., Carraresi, C., Stopani, E., \& Bulli, F. (2015b). The two dimensions of contamination fear in obsessive-compulsive disorder: Harm avoidance and disgust avoidance. Journal of Obsessive-Compulsive and Related Disorders, 6, 124-131. 
Melli, G., Chiorri, C., \& Smurra, R. (2013). Further revision and psychometric properties of an Italian version of the «Disgust Scale-Revised». Psicoterapia Cognitiva e Comportamentale, 19, 63-93.

Melli, G., Chiorri, C., Stopani, E., Bulli, F., \& Carraresi, C. (2017a). Development and validation of a new short measure of disgust propensity: the Disgust Propensity Questionnaire (DPQ). Clinical Psychology \& Psychotherapy, 24, 1189-1204.

Melli, G., Gremigni, P., Elwood, L. S., Stopani, E., Bulli, F., \& Carraresi, C. (2015c). The Relationship Between Trait Guilt, Disgust Propensity, and Contamination Fear. International Journal of Cognitive Therapy, 8, 1-13.

Melli, G., Moulding, R., \& Poli, A. (2016). Is it more disgusting if I think about it? Examining the interaction of obsessive beliefs and disgust propensity in clinical obsessive-compulsive disorder. Journal of Obsessive-Compulsive and Related Disorders, 11, 74-81.

Menard, S. (1995). Applied logistic regression analysis. Sage University paper series on quantitative applications in the social sciences, 07-106. Thousands Oaks, CA: Sage.

Miron-Shatz, T., Stone, A., \& Kahneman, D. (2009). Memories of yesterday's emotions: Does the valence of experience affect the memory-experience gap? Emotion, 9(6), 885-891. https://doi.org/10.1037/a0017823

Moretz, M. W., \& McKay, D. (2008). Disgust sensitivity as a predictor of obsessive-compulsive contamination symptoms and associated cognitions. Journal of anxiety disorders, 22, 707-715.

Olatunji, B. O. (2010). Changes in disgust correspond with changes in symptoms of contaminationbased OCD: A prospective examination of specificity. Journal of Anxiety Disorders, 24, 313317.

Olatunji, B. O. (2015). Selective effects of excessive engagement in health-related behaviors on disgust propensity. Cognition and Emotion, 29, 882-899. 
Olatunji, B. O., Ebesutani, C., David, B., Fan, Q., McGrath, P. B. (2011a). Disgust proneness and obsessive-compulsive symptoms in a clinical sample: Structural differentiation from negative affect. Journal of Anxiety Disorders, 25, 932-938.

Olatunji, B. O., Sawchuk, C. N., Lohr, J. M., \& de Jong, P. J. (2004). Disgust domains in the prediction of fear of contamination. Behaviour Research and Therapy, 42, 93-104.

Olatunji, B. O., Tart, C. D., Ciesielski, B. G., McGrath, P. B., \& Smits, J. A. J. (2011b). Specificity of disgust vulnerability in the distinction and treatment of OCD. Journal of Psychiatric Research, 45, 1236-1242.

Olatunji, B. O., Williams, N. L., Lohr, J. M., \& Sawchuk, C. N. (2005). The structure of disgust: Domain specificity in relation to contamination ideation and excessive washing. Behaviour Research and Therapy, 43, 1069-1086.

Olatunji, B. O., Williams, N. L., Tolin, D. F., Abramowitz, J. S., Sawchuk, C. N., Lohr, J. M., \& Elwood, L. S. (2007). The Disgust Scale: Item analysis, factor structure, and suggestions for refinement. Psychological Assessment, 19, 281-297.

Power, M., \& Dalgleish, T. (1997). Cognition and emotion: From order to disorder. East Sussex, UK: Psychology Press.

Rachman, S. J. (2004). Fear of contamination. Behaviour Research and Therapy, 42, 1227-1255.

Radomsky, A. S., Rachman, S. J., Shafran, R., Coughtrey, A. E., \& Barber, K. C. (2014). The nature and assessment of mental contamination: A psychometric analysis. Journal of ObsessiveCompulsive and Related Disorders, 3, 181-187.

Redelmeier, D. A., Katz, J., \& Kahneman, D. (2003). Memories of colonoscopy: A randomized trial. Pain, 104(1-2), 187-194. https://doi.org/10.1016/S0304-3959(03)00003-4

Rouel, M., Stevenson, R., \& Smith, E. (2018). Predicting Contamination Aversion Using Implicit and Explicit Measures of Disgust and Threat Overestimation. Behaviour Change, 1-17. HTTP://DX.DOI.ORG/ 10.1017/bec.2018.6 
Safer, M. A., \& Keuler, D. J. (2002). Individual differences in misremembering pre-psychotherapy distress: personality and memory distortion. Emotion, 2, 162-178. http://dx.doi.org/10.1037/1528-3542.2.2.162

Schienle, A., Stark, R., Walter, B., \& Vaitl, D. (2003). The connection between disgust sensitivity and blood-related fears faintness symptoms and obsessive-compulsiveness in a non-clinical sample. Anxiety Stress and Coping, 16, 185-193.

Schwarz, N. (1999). Self-reports: How the questions shape the answers. American Psychologist, 54(2), 93-105. http://dx.doi.org/10.1037/0003-066X.54.2.93

Schwarz, N. (2007). Retrospective and concurrent self-reports: The rationale for real-time data capture. In A. Stone, S. S. Shiffman, A. Atienza, \& L. Nebeling (eds.) (2007). The science of real-time data capture: Self-reports in health research (pp. 11-26). New York: Oxford University Press.

Sica, C., \& Ghisi, M. (2007). The Italian versions of the Beck Anxiety Inventory and the Beck Depression Inventory-II: Psychometric properties and discriminant power. In M. A. Lange (Ed.), Leading-Edge Psychological Tests and Testing Research (pp. 27-50). Hauppauge, New York: NOVA Publishers.

Storch, E. A., Rasmussen, S. A., Price, L. H., Larson, M. J., Murphy, T. K., \& Goodman, W. K. (2010). Development and psychometric evaluation of the Yale-Brown Obsessive-Compulsive Scale - Second Edition. Psychological Assessment, 22, 223-232.

Stice, E. (2002). Risk and Maintenance Factors for Eating Pathology: A Meta-Analytic Review. Psychological Bulletin, 128, 825-848.

Stone, A.A., Shiffman, S. S., \& DeVries, M. W. (1999). Ecological momentary assessment. In D. Kahneman, E. Diener, \& N. Schwarz (Eds.), Well-being: The foundations of hedonic psychology (pp. 61-84). New York: Russell-Sage

Sudman, S., Bradburn, N. M., \& Schwarz, N. (1996). Thinking about answers: The application of cognitive processes to survey methodology. San Francisco, CA: Jossey-Bass. 
Summerfeldt, L., Antony, M. M., Downie, F., Richter, M. A., \& Swinson, R. P. (1997). Prevalence of particular obsession and compulsions in a clinic sample. Unpublished manuscript.

Thomas, D. L., \& Diener, E. (1990). Memory accuracy in the recall of emotions. Journal of Personality and Social Psychology, 59(2), 291-297. http://dx.doi.org/10.1037/00223514.59.2.291

Thorpe, S. J., Patel, S. P., \& Simonds, L. M. (2003). The relationship between disgust sensitivity, anxiety and obsessions. Behaviour Research and Therapy, 41, 1397-1409.

Tolin, D. F., Woods, C. M., \& Abramowitz, J. S. (2006). Disgust sensitivity and obsessivecompulsive symptoms in a non-clinical sample. Journal of Behavior Therapy and Experimental Psychiatry, 37, 30-40.

Van den Bergh, O., \& Walentynowicz, M. (2016). Accuracy and bias in retrospective symptom reporting. Current Opinion in Psychiatry, 29(5), 302-308. http://dx.doi.org/10.1097/YCO.0000000000000267

Woody, S. R., \& Teachman, B. A. (2000). Intersection of disgust and fear: Normative and pathological views. Clinical Psychology: Science and Practice, 7, 291-311.

Woody, S. R., \& Tolin, D. F. (2002). The relationship between disgust sensitivity and avoidant behavior: Studies of clinical and nonclinical samples. Journal of Anxiety Disorders, 16, 543-555. 
Heightened disgust propensity and contamination fear in OCD - 24

Table 1. Demographic data across the two groups.

\begin{tabular}{llcc}
\cline { 2 - 3 } Variable & Category & $\begin{array}{c}\text { C-OCD } \\
(n=56)\end{array}$ & $\begin{array}{c}\text { NC-OCD } \\
(n=103)\end{array}$ \\
\hline Gender $(\mathrm{P})$ & Female & $54 \%$ & $70 \%$ \\
& Male & $46 \%$ & $30 \%$ \\
Age $(\mathrm{M} \pm \mathrm{SD})$ & & $35.20 \pm 9.50$ & $34.00 \pm 9.40$ \\
Years of education $(\mathrm{M} \pm \mathrm{SD})$ & & & \\
& & $14.67 \pm 4.04$ & $14.08 \pm 3.14$ \\
Marital Status $(\mathrm{P})$ & Single & $64 \%$ & $63 \%$ \\
& Married & $34 \%$ & $32 \%$ \\
& Divorced & $2 \%$ & $5 \%$ \\
Occupation $(\mathrm{P})$ & & & \\
& Housemaker & $4 \%$ & $2 \%$ \\
& Employee & $25 \%$ & $38 \%$ \\
& Professional & $20 \%$ & $15 \%$ \\
& Unoccupied & $18 \%$ & $6 \%$ \\
& Student & $18 \%$ & $28 \%$ \\
& Other & $15 \%$ & $11 \%$ \\
\hline
\end{tabular}

Note: $\mathrm{C}-\mathrm{OCD}=$ Contamination-related Obsessive-Compulsive Disorder; $\mathrm{NC}-\mathrm{OCD}=$ Non contamination-related Obsessive-Compulsive Disorder; $\mathrm{P}=$ proportion; $M=$ Mean; $S D=$ Standard Deviation. 
Heightened disgust propensity and contamination fear in OCD - 25

Table 2. Mean and standard deviations for DPQ scores between and within groups.

\begin{tabular}{lcc}
\hline & DPQ-T0 & DPQ-T1 \\
\hline C-OCD & $13.20(7.99)$ & $21.37(8.15)$ \\
NC-OCD & $13.10(8.18)$ & $13.02(7.48)$ \\
\hline
\end{tabular}

Note: $\mathrm{C}-\mathrm{OCD}=$ Contamination-related Obsessive-Compulsive Disorder; NC-OCD $=$ Non contamination-related Obsessive-Compulsive Disorder; $\mathrm{M}(\mathrm{SD})=$ Mean score $-\mathrm{SD}=$ Standard Deviation in parentheses; DPQ-T1 = Disgust Propensity Questionnaire - current; DPQ-T0 = Disgust Propensity Questionnaire - in reference to 18 years old of age. 
Table 3. Pearson's correlations in the whole sample $(n=159)$.

\begin{tabular}{llllllll}
\hline Measure & 1 & 2 & 3 & 4 & 5 & 6 & 7 \\
\hline 1. DPQ-T1 & - & & & & & & \\
2. DPQ-T0 & $.58^{* *}$ & - & & & & & \\
3. DOCS-Contamination & $.53^{* *}$ & .06 & - & & & & \\
4. DOCS-Responsibility & .09 & $.18^{*}$ & .09 & - & & & \\
5. DOCS-Unacceptable Thoughts & $-.23^{*}$ & .10 & $-.26^{* *}$ & $.24 * *$ & - & & \\
6. DOCS-Symmetry & $.24^{*}$ & $.21^{*}$ & $.19^{*}$ & $.41^{* *}$ & $.22 * *$ & - & \\
7. BDI-II & $.19^{*}$ & $.19^{*}$ & .10 & $.29^{* *}$ & $.31^{* *}$ & $.22^{* *}$ & - \\
8. BAI & .12 & $.20^{*}$ & .07 & $.31^{* *}$ & $.38^{* *}$ & $.27^{* *}$ & $.69^{* *}$ \\
\hline
\end{tabular}

Note: DPQ-T1 = Disgust Propensity Questionnaire - current; DPQ-T0 = Disgust Propensity Questionnaire -18 years old of age; DOCS = Dimensional Obsessive-Compulsive Scale; BDI-II = Beck Depression Inventory-II; BAI = Beck Anxiety Inventory;

$* * p<.001$

$* p<.05$ 
Table 4. Hierarchical multiple regression analyses in the C-OCD sample $(n=56)$.

\begin{tabular}{lll}
\hline Predictor & Model 1 & Model 2 \\
\hline \multicolumn{2}{l}{ Criterion: DOCS Contamination } & \\
\hline$\Delta R^{2}$ & .01 & $.27^{*}$ \\
BDI-II & $.09(.06)$ & $-.01(.05)$ \\
BAI & $.01(.06)$ & $.01(.06)$ \\
DPQ-T1 & $.53(.05)^{*}$ \\
Criterion: DOCS Contamination & \\
\hline$R^{2}$ & .01 & .01 \\
BDI-II & $.09(.06)$ & $.09(.06)$ \\
BAI & $.01(.06)$ & $.01(.07)$ \\
DPQ-T0 & & $.04(.07)$ \\
\hline
\end{tabular}

Note: Standard errors in parentheses; DOCS = Dimensional Obsessive-Compulsive Scale; BDI-II = Beck Depression Inventory-II; BAI = Beck Anxiety Inventory; DPQ-T1 = Disgust Propensity Questionnaire - current; DPQ-T0 = Disgust Propensity Questionnaire -18 years old of age.

$* p<.001$ 